\title{
An Enhanced Approach for Classification in Web Usage Mining using Neural Network Learning Algorithms for Supervised Learning
}

\author{
Jaykumar Jagani \\ R.K. University \\ R.K. University, Kasturbadham, \\ Near Tramba, Rajkot - 360020
}

\author{
Kamlesh patel \\ Professor, R.K. University \\ R.K. University, Kasturbadham, \\ Near Tramba, Rajkot - 360020
}

\begin{abstract}
Now a day data on the web is growing by a rapid speed, large volume of data is available on web. So, extract useful knowledge from large web data, efficient web mining methods are required to handle those data and achieve various functionalities such a as user trend analysis, web profile analysis, Web AD market change analysis, etc. The concept of neural network helps to handle large volume of data by its characteristics [1]. Several neural network learning algorithms provides better supervised learning. They are capable to handle huge dynamic data. Specially, LVQ (Learning Vector Quantization) algorithms are useful for supervised, dynamic labeling or post-training map labeling and supervised version of SOM through that the approximation of distribution of class with less number of codebook vectors and able to minimize classification errors respectively [9]. MLVQ and HLVQ are both the techniques are following a concept of multi pass in which more than one pass can be performed on the same model and is very useful for gaining best desired results [11]. Here in this paper, we are going to discuss a new technique that will work on hierarchical as well as multi pass approach that is having the advantages of both multi pass and hierarchical approach by combining the benefits of both and designed a new algorithm. That new technique will more accurate, less time consuming and able to decrease learning rate for neural network. The basic HLVQ approach will follow the same algorithm for generation of all phases. In addition to that HMLVQ provides better efficiency through enhancing advantages the various approaches for the same classification process.
\end{abstract}

\section{General Terms}

Web Usage Mining, Clustering and Artificial Neural Networks.

\section{Keywords}

LVQ, MLVQ, HLVQ, Web log data, classification.

\section{INTRODUCTION}

Web users are increasing day by day so the web data on the web is growing more. [6] Now, any mega firms or IT companies want to survive in the market, so they need to analyze the current web data and historical web data as well as the existing trends of current generation users and prediction of future trends. All these are possible only by analyzing web data. Various data mining techniques are applied on huge web data to extract useful knowledge for decision making by business Analysts [3].

As there are many web scaling problems such as user trend analysis of surfing, traffic flow analysis, distributed control handling, web traffic management and many more. Session tracking and website reorganization, distributed traffic sharing on distributed servers can be identified and analysis based on web data can be possible through web mining. But for that the large amount of rough data the accurate classification is required and that classification can be possible using concepts of neural network [1] [4] [6] [2].

In this Paper, the discussion is based on various neural network learning algorithms that help to handle large web data as well as better classification and clustering of data with less number of errors. Self-Organizing Maps called SOM and Learning Vector Quantization known as LVQ are very constructive learning algorithms that classify and cluster the web data successfully [9]. SOM are useful for supervised, unsupervised learning, dynamic labeling or post-training map labeling. Learning vector quantization is useful for the approximation of distribution of class with less number of codebook vectors and able to minimize classification errors [9]. MLVQ and HLVQ techniques are following a concept of multi pass in which more than one pass can be performed on the same model using different algorithms and are very useful for gaining best preferred results. So in this paper, the discussion is based on a new technique that will work on multi pass as well as on hierarchical approach. So the advantages of both techniques will be achieved in single algorithm.

\section{WEB USAGE MINING}

Web Usage Mining in a simple term is to extract the usage knowledge about the web users through various data mining techniques from web data is web usage mining [4]. Web Usage Mining is going to prove more useful method in current and next generation's web exploring people world [6]. So, there is a huge research scope available in this area to develop and implement new ideas that how Usage Mining can be more effective and as a result of that end user is able to get better facilities as developer firms will able to identify user's interest and will able to deliver the products likewise.

\subsection{Sources of data for Web Usage Mining}

The data input for the various classification algorithms can be found from (1) Web servers (2) Proxy Servers (3) Web Clients [3]. The data is stored on the web in web log files. Each and every user activity is stored in respective server. The data is fetched from that server for analysis. The web data available on the server is in a specific format known as extended common log format (ECLF) [5].

ECLF Format 
Table 1 (various columns of ECLF format)

\begin{tabular}{|l|l|l|l|l|}
\hline I/P & Rfc 931 & Auth User & $\begin{array}{l}\text { Req. Time } \\
\text { Stamp }\end{array}$ & $\begin{array}{l}\text { Req. } \\
\text { Format }\end{array}$ \\
\hline Status & Bytes & Referrer & $\begin{array}{l}\text { User } \\
\text { Agent }\end{array}$ & \\
\hline
\end{tabular}

Important terms

Ipaddress- network address of user machine

Rfc 931- remote login name of user

Status- as success / page not found like errors

User agent- software or browser (web client)

Authuser - original user name

Bytes - size of transferred information [6]

\subsection{Process of Web Usage Mining}

As per Figure 1, it is clear that process of web usage mining is much similar to data mining process.

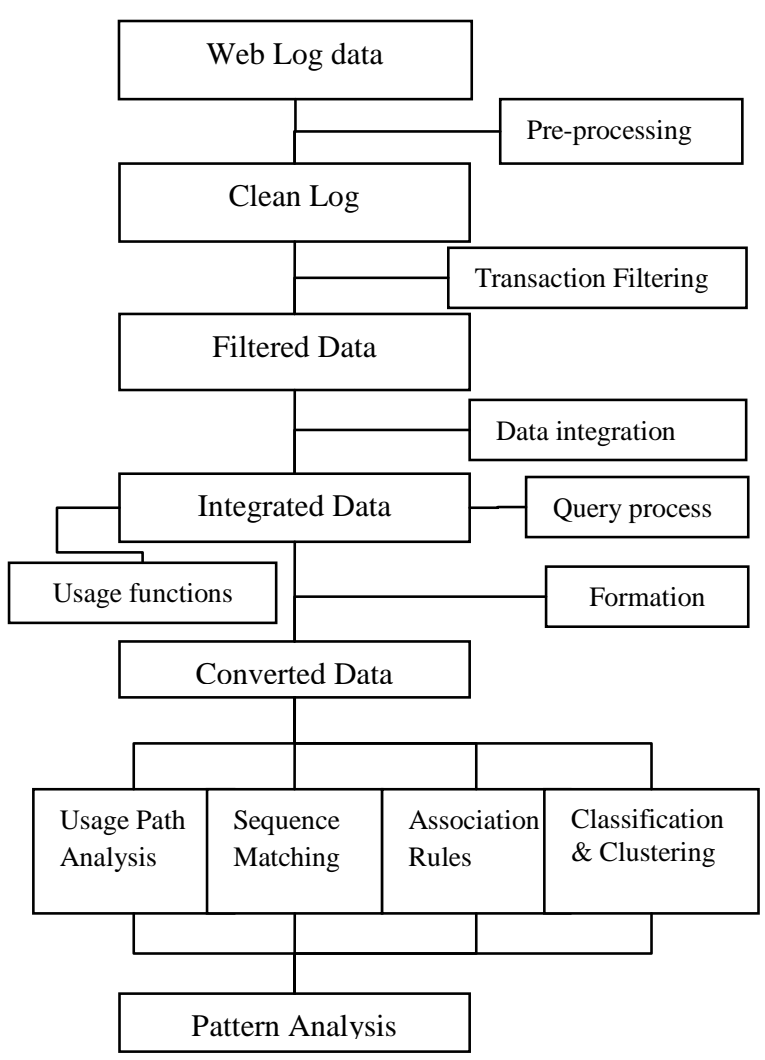

Figure-1 Common Process of Web Usage Mining

As per figure 1, The Only difference in data mining and web mining is that at the Starting level in data will come from various data bases, warehouses, and flat files and in web mining data will come from server log files. In this Paper, the focus is on initial phase of web usage mining process where clustering and classification process are done to provide better extraction and stability of data.

\section{ROLE OF NEURAL NETWORK FOR WEB USAGE MINING}

Neural network provides many supervised learning algorithms that can be helpful in mining process, especially to give class labels to uncategorized data i.e. classification. Here is some brief about neural networks and its useful characteristics. The network of highly connected, self-intelligent neurons (Nodes) to do any task with once training is neural network.

\subsection{Artificial Neural Network in brief}

Artificial neural network (ANN) is a knowledge processing paradigm that inspired from biological nervous system. This technology is more useful because of its unique structure of system. ANN is the arrangement of large number of highly connected processing elements, called neurons in medical science as in brain, working together to solve a particular problem [1]. ANN is configured i.e. initializes with training or testing data to solve a particular problem such as, clustering or classification. Neural networks are highly capable to do the things such as meaning derivation from complex data. It is mostly used in finding usage trends that are sometimes very common but complex and even not carried out by machines.

Neural network has many advantages as follows:

- Self-adaptive learning

How to deal with problems based on initial training or experience from the network.

- Self-organization

Artificial neural network creates its own organization and behavior and representation of knowledge it accepts while learning.

- $\quad$ Real time operations

Neural network computation may carry out parallel but for that special hardware are required to design.

- $\quad$ Fault tolerance via multiple information copies Partial destroys or failure of network cannot affect the performance of the network [6].

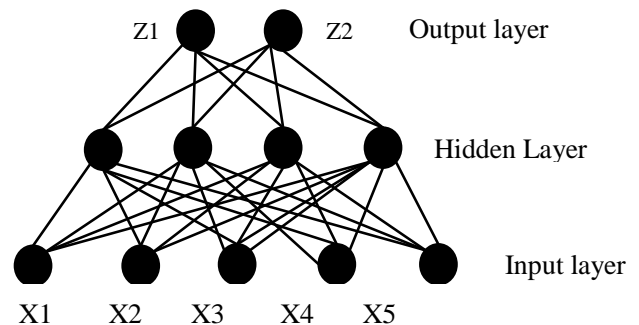

Figure-2 Processing Architecture of Neural Network

As figure 2, shows the recurrent network of ANN map nodes with its all three layers.

\section{NEURAL NETWORK ALGORITHMS FOR CLASSIFICATION IN WEB USAGE MINING}

The list of such algorithm is big, but in this paper, discussion on some of the best classifiers among them, described in following sections. 


\subsection{Learning Vector Quantization (LVQ)}

It is a machine learning algorithm and supervised version of SOM Algorithm. Through LVQ the approximation of distribution of class with less number of codebook vectors and is able to minimize classification errors. Codebook vectors are considered as class boundary representations. The algorithm is associated with neural network of class learning algorithms [8].

\subsubsection{Advantages of $L V Q$}

LVQ is capable to summarize large datasets to smaller number of codebook vectors that are useful for visualization and classification. Training rate is so fast compare to any other network like back propagation. The Generated model updated incrementally. Here some LVQ versions are available.

\subsection{Versions of LVQ}

They are denoted as LVQ1, LVQ2, LVQ3, OLVQ1, OLVQ3, MLVQ and hierarchical LVQ [11].

\subsubsection{LVQ1}

Imagine that a number of 'codebook vectors' cvi (free bound vectors) are located into the input gap to estimate various domains of the input vector ' $v$ ' by their quantized values [8]. Generally a number of codebook vectors are allocated to each class of $\mathrm{v}$ values, and $\mathrm{v}$ is then decided to fit in to the same class to which the adjacent cvi belongs.

Let $\boldsymbol{d}=\arg \min (\|\boldsymbol{v}-\boldsymbol{c v} \boldsymbol{i}\|)$ describe the nearest cvi to $\mathrm{v}$, denoted by dc.

Standards for cvi that just about reduce the misclassification errors in the over nearest-neighbor classification can be found as asymptotic values in the subsequent learning process. Let $\mathrm{v}(\mathrm{t})$ be a sample of input and let the cvi(t) stand for sequences of the cvi in the discrete-time domain. Beginning with properly defined initial values, the following equations define LVQ1.

$d c(t+1)=d c(t)+\operatorname{alpha}(t)[v(t)-d c(t)]$

If $\mathrm{v}$ and dc belong to the similar class,

$d c(t+1)=\operatorname{dc}(t)-\operatorname{alpha}(t)[v(t)-d c(t)]$

If $\mathrm{x}$ and $\mathrm{mc}$ belong to dissimilar classes,

$\operatorname{cvi}(t+1)=\operatorname{cvi}(t)$, for I is not in $\mathrm{c}$.

Here $0<$ alpha $(\mathrm{t})<1$, and alpha $(\mathrm{t})$ may be steady or decrease monotonically with time. In the above basic LVQ1 it is recommended that alpha should initially be lesser than 0.1 ; linear decrement in time is used in this package. [8]

\subsection{2 $L V Q 2$}

The classification verdict in this algorithm is the same with that of the LVQ1. In learning, however, two codebook vectors, cvi and cvj that are the adjoining neighbors to $\mathrm{v}$, are now updated concurrently [7]. One of them must be in the right place to the correct class and the other to a wrong class, correspondingly. Moreover, $v$ must fall into a region of values called 'window', which is defined around the mid plane of cvi and cvj. Assume that edi and edj are the Euclidean distances of v from cvi \&cvj, correspondingly [7]; then v is defined to fall in a 'window' of qualified width $w$ if, $\min \left(\frac{e d i}{e d j}, \frac{e d j}{e d i}\right)>S$, where $s=(1-w) /(1+w)$

Width w of the 'window' should be 0.2 to 0.3

$$
\begin{aligned}
& \text { 4.2.2.1 Algorithm } \\
& \operatorname{cvi}(t+1)=\operatorname{cvi}(t)-\operatorname{alpha}(t)[v(t)-\operatorname{cvi}(t)], \\
& \operatorname{cvj}(t+1)=\operatorname{cvj}(t)+\operatorname{alpha}(t)[v(t)-\operatorname{cvj}(t)]
\end{aligned}
$$

Where cvi and cvj are the two closest codebook vectors to v and whereby $\mathrm{v}$ and $\mathrm{cvj}$ belong to the same class, while $\mathrm{v}$ and cvi belong to different classes, respectively [7]. Furthermore v must fall into the 'window'.

\subsubsection{LVQ3}

The LVQ2 algorithm was based on the thought of differentially changing the decision limitations towards the Bayes restrictions, while no notice was paid to what might has done to the location of the min the extended run if this process were continued. As a result it seems like required having corrections that make sure that the cvi continue resembling the class distributions, at least more or less. Combining these ideas, we now acquire an improved algorithm that is called LVQ3 [9].

As per Equation 4,

$$
m k(t+1)=m k(t)+\text { epsilon alpha }(t)[v(t)-m k(t)]
$$

\subsubsection{The Optimized LVQ (OLVQ1)}

The basic LVQ1 algorithm is now modified in such a way that an individual learning rate alpha $(t)$ is assigned to each cvi [7]. We then get the following discrete time learning process. Let $\mathrm{c}$ be defined by Eq. (1). Then,

$$
d c(t+1)=d c(t)+\operatorname{alpha}(t)[v(t)-d c(t)]
$$$$
\text { If } \mathrm{v} \text { is classified correctly, }
$$

$$
d c(t+1)=\operatorname{dc}(t)-\operatorname{alpha}(t)[v(t)-d c(t)]
$$

If the classification of $\mathrm{v}$ is incorrect,

$\operatorname{cvi}(t+1)=\operatorname{cvi}(t)$, for i not in c.

We tackle the problem irrespective of the alphai $(t)$ can be firmed optimally for fastest possible convergence of eq. (6) [7]. If we state (6) in the form,

$$
\begin{array}{r}
d c(t+1)=[1-s(t) \operatorname{alphac}(t)] d c(t)+\underset{s(t) \operatorname{alphac}(t) v(t)}{+}
\end{array}
$$

Where $s(t)=+1$ or -1 if the classification is accurate and incorrect respectively, we primary straight see that $d c(t)$ is statistically liberated from $v(t)$. It may also be noticeable that the statistical correctness of the learned codebook vector values is optimal if the assets of the corrections made at different times, when referring to the end of the learning period, are of the same weight. Notice that $d c(t+1)$ contains a "trace" from $v(t)$ through the last term in (7), and "traces" from the earlier $v\left(t^{\prime}\right) ; t^{\prime}=1,2, \ldots t-1$ through $d c(t)$. The magnitude of the last "trace" from $v(t)$ is scaled down by the factor alphac $(t)$, and, for instance, the "outline" from $x(t-1)$ is scaled down by $[1-(t) \operatorname{alphac}(t)]$ $\operatorname{alphac}(t-1)[7]$. 
Now we first specify that these two scaling must be identical:

$\operatorname{alphac}(t)=[1-s(t) \operatorname{alphac}(t)] \operatorname{alphac}(t-1)$

If this condition is then made to hold for all $t$, by induction it can be shown that the "traces" collected up to time $t$ from all the earlier $\mathrm{x}$ will be scaled down by an equal amount at the end, and thus the "optimal" values of alphai $(t)$ are determined by the recursion,

$$
\operatorname{alphac}(t)=\operatorname{alphac}(t-1) /(1+s(t) \operatorname{alphac}(t-1))
$$

\subsubsection{Multi pass $L V Q$}

It is like supervised version of multi pass SOM where quick rough pass can be made on the model by using OLVQ1 algorithm and then the long fine tuning pass can be made on the model through any of LVQ1, LVQ 2 or LVQ3 [11].

\subsubsection{Hierarchical $L V Q$}

Here an LVQ model is constructed and each codebook vector is treated as a cluster centroid. All codebook vectors are evaluated and numbers are selected as candidates for sub-

\subsubsection{LVQ Algorithm Comparison}

models. Sub models are constructed for all candidate codebook vectors and those sub models that outperform (in terms of classification accuracy) their parent codebook vector are kept as part of the model [10]. During testing a data instance is first mapped onto its BMU, if that BMU has a submodel (that was not pruned during training), the sub model is used for classification, otherwise the class value in the BMU is used for classification. This algorithm has proven most useful for large datasets where a low-complexity model is used as the base model, and specialized LVQ models are used at each codebook vector to better model the data [10].

\subsubsection{General considerations and Comparison}

In LVQ, vector quantization is not worn to estimate to density functions of the class samples, but to unswervingly define the class borders along with the nearest-neighbor rule. The accuracy reachable in any classification task to which the LVQ algorithms are useful and the time needed for learning depend on the subsequent factors:

An approximately most favorable number of codebook vectors assigned to each class and their initial values, the comprehensive algorithm, a proper learning rate applied during the steps, and a proper condition for the stopping of learning.

Table 2: All LVQ algorithm comparison with respect to various fields

\begin{tabular}{|l|l|l|l|l|}
\hline Fields & LVQ & OLVQ & MLVQ & HLVQ \\
\hline Version & Basic & Optimized & Multiple passes & Hierarchical \\
\hline Accuracy & Less & More than LVQ & More than OLVQ & More than MLVQ \\
\hline Time & High & Less than LVQ & Less than OLVQ & Less than MLVQ \\
\hline Efficiency & Low & Medium & High & High \\
\hline Codebooks & More & Moderate & Less & Less \\
\hline Capacity & Low & Medium & High & High \\
\hline
\end{tabular}

\section{PROPOSED WORK}

After discussing to the very basic concepts and algorithms of neural network learning algorithms, now discussion is how the accuracy of algorithms can be enhanced. Now, we are proposing a new technique with the combination of HLVQ and MLVQ approach that is known as HMLVQ.

\subsection{Basic HLVQ Approach}

Here defined the steps for basics HLVQ:

Here, HLVQ is divided into two Stages like A is $1^{\text {st }}$ and B is $2^{\text {nd }}[10]$.
Table 3: Basic HLVQ algorithm [10]

\begin{tabular}{|c|c|}
\hline 1. & Initialize the codebook vectors $\mathrm{Wi}$ and the learning \\
\hline 2. & Randomly select an input vector $\mathrm{X} 1$ \\
\hline 3. & Find the winner unit closest to $\mathrm{X} 1$ by considering \\
\hline \multirow[t]{3}{*}{3.1} & Modify the weights of the winner unit: \\
\hline & $\begin{array}{l}\text { If } W c \text { and } X \text { belong to the same class } \\
\qquad W c(t+1)=W c(t)+\alpha(t)[X(t)-W c(t)]\end{array}$ \\
\hline & $\begin{array}{l}\text { If } W c \text { and } X \text { belong to different classes } \\
\quad W c(t+1)=W c(t)-\alpha(t)[X(t)-W c(t)] .\end{array}$ \\
\hline 4. & $\begin{array}{l}\text { Repeat from step } 2 \text { until the neural network } \mathrm{C} \text { is } \\
\text { stabilized or until a fixed number of iterations have } \\
\text { been carried out. }\end{array}$ \\
\hline 5. & Initialize the codebook vectors $W j$ for $\mathrm{B}$. \\
\hline 6. & Randomly select an input vector X2 \\
\hline 7. & Repeat Step 3 and 3.1 \\
\hline 8. & $\begin{array}{l}\text { Reduce learning Rate of } \propto \& \text { repeat step } 5 \text { until } \\
\text { fixed steps. }\end{array}$ \\
\hline
\end{tabular}




\subsection{Hierarchical Multi pass LVQ}

As it is obvious that multi pass and hierarchical LVQs are having advantages such as multi pass LVQ is a hybrid of basic and optimized LVQ so it has advantages of all basic LVQs and it works in the pass, so speed achieved for even more learning data is more and Hierarchical LVQ gives accuracy as it works in detail with domains and sub domains i.e. hierarchy levels also.

Now if any technique has the advantages of both multi pass LVQ and Hierarchical LVQ, then it would be very good to classify with it any large data within efficient time. Such method is proposed as Hierarchical Multi pass LVQ. It has advantage of both multi pass and hierarchical technique that is speed and accuracy both. So, classification errors are reduced and process speed is also increased that means the large raw data can be classified in sufficient amount of time with more accuracy.

\subsubsection{Proposed steps of $H M L V Q$}

Here we are proposing the steps for HMLVQ:

Here $i$ is an input vector, $\mathrm{C}$ is codebook vector, and $\propto$ is learning rate and $\mathrm{N}$ is Neural Network.

\section{Table 4: Proposed HMLVQ algorithm}

\begin{tabular}{|l|l|}
\hline 1. & $\begin{array}{l}\text { Initialize the codebook vectors } \mathrm{C} \text { with fixed learning } \\
\text { rate } \alpha \text { for first stage. }\end{array}$ \\
\hline 2. & $\begin{array}{l}\text { Give an input vector I and find the closest unit } \\
\text { nearby vector } \mathrm{C} \text { using Euclidian distance between }\end{array}$ \\
\hline 3. & Update the weights of closest unit \\
\hline 3.1 & $\begin{array}{l}\text { If they are in same class then unit weight is added in } \\
\text { classification results and result is correct. } \\
\mathrm{Ci}(t+1)=C i(t)+\propto(t)[I(t)-C i(t)] . \\
\text { they are not in same class then unit weight is } \\
\text { subtracted and result is not accurate. } \\
C i(t+1)=C i(t)-\propto(t)[I(t)-C i(t)] .\end{array}$ \\
\hline 4. & $\begin{array}{l}\text { Repeat from step } 2 \text { until the neural network } \mathrm{N} \text { is } \\
\text { stabilized with fixed number of iterations. }\end{array}$ \\
\hline 5. & Reduce learning rate at end of each level. \\
\hline 6. & $\begin{array}{l}\text { Repeat the same procedure for Second Stage by } \\
\text { considering the best result amongst the current stage } \\
\text { and all previous stage. }\end{array}$ \\
\hline 7. & Select most accurate unit and discard all rest. \\
\hline 8. & $\begin{array}{l}\text { Repeat the procedure until the whole dataset is } \\
\text { classified. }\end{array}$ \\
\hline
\end{tabular}

\subsubsection{Proposed working of $H M L V Q$}

For HMLVQ, an LVQ model is constructed using multi pass and each codebook vector is treated as a cluster centroid. All codebook vectors are evaluated and numbers are selected as candidates for sub-models. Sub models are also constructed using multi pass for all candidate codebook vectors and those sub models that do not perform well (in terms of classification accuracy), their parent codebook vector are kept as participant of the model. During test phase a data instance is first mapped to its BMU, if that BMU has a sub-model (that was not pruned during training), the sub model is used for classification, if that was pruned during training; the class value in the BMU is used for classification.

So In short at each level the model is constructed using multi pass LVQ and codebook vectors are evaluated and given numbers as candidate for sub model. The sub model is also constructed using multi pass LVQ specially OLVQ1. So, at each and every hierarchy level the sub models are constructed using multi pass LVQ if sub model is more inaccurate then it is rejected and parent node is considered as an element in model. By using this approach accuracy maintains at each and every level and construction of sub models are also fast and accurate.

\subsubsection{Comparison of Both HLVQ and HMLVQ}

\begin{tabular}{|c|c|}
\hline Basic Approach & My approach HMLVQ \\
\hline $\begin{array}{l}\text { It is basic hierarchical } \\
\text { approach with one } \\
\text { algorithms for all time } \\
\text { i.e. lvq3 }\end{array}$ & $\begin{array}{l}\text { Hierarchical approach with } \\
\text { different algorithms for all } \\
\text { stage classification }\end{array}$ \\
\hline $\begin{array}{l}\text { Parameters: Accuracy, } \\
\text { Time }\end{array}$ & Parameters: Accuracy, Time \\
\hline \multicolumn{2}{|c|}{ Available Algorithms } \\
\hline \multicolumn{2}{|c|}{ For Initial Time: LVQ1,LVQ2, LVQ3, OLVQ1,OLVQ3 } \\
\hline \multicolumn{2}{|c|}{ For Next Rounds (if exists): MLVQ, OLVQ1,OLVQ3 } \\
\hline For first stage generation & For first stage generation \\
\hline Take Any one Approach & Take the Same Approach \\
\hline e.g. OLVQ1 & e.g. OLVQ1 \\
\hline $\begin{array}{l}\text { For Second stage } \\
\text { generation }\end{array}$ & For Second stage generation \\
\hline Took the same Approach & $\begin{array}{l}\text { Here I changed Approach I } \\
\text { took }\end{array}$ \\
\hline e.g. OLVQ1 & MLVQ \\
\hline \multirow[t]{2}{*}{ Continues same for rest } & Continues same for rest of \\
\hline & Reduce learning Rate \\
\hline \multicolumn{2}{|c|}{ Result is benefit in accuracy and Time } \\
\hline
\end{tabular}

\subsubsection{Simulation of $H M L V Q$}

Here I have tried some simulation based on my approach and I have found the new results comparatively better than old approach with increase in efficiency with less execution time. I have taken sample data and compare both the approaches. Results are as follows gives the clear cut idea of it.

Data samples are:

- $\quad$ Breast Cancer(BC)

- $\quad$ Super Market(SM)

- $\quad$ Log data(LOG)

Table 6: Simulation HLVQ vs. HMLVQ

\begin{tabular}{|l|l|l|l|l|}
\hline Field & \multicolumn{3}{l|}{ Datasets } \\
\hline Algorithm & Params & BC & SM & LOG \\
\hline HLVQ & Accuracy & 93.71 & 64.88 & 80.33 \\
\hline & Time & $13 \mathrm{~ms}$ & $64 \mathrm{~ms}$ & $12 \mathrm{~ms}$ \\
\hline & Accuracy & 95.46 & 66.80 & 80.48 \\
\hline HMLVQ & Time & $5 \mathrm{~ms}$ & $63 \mathrm{~ms}$ & $11 \mathrm{~ms}$ \\
\hline
\end{tabular}




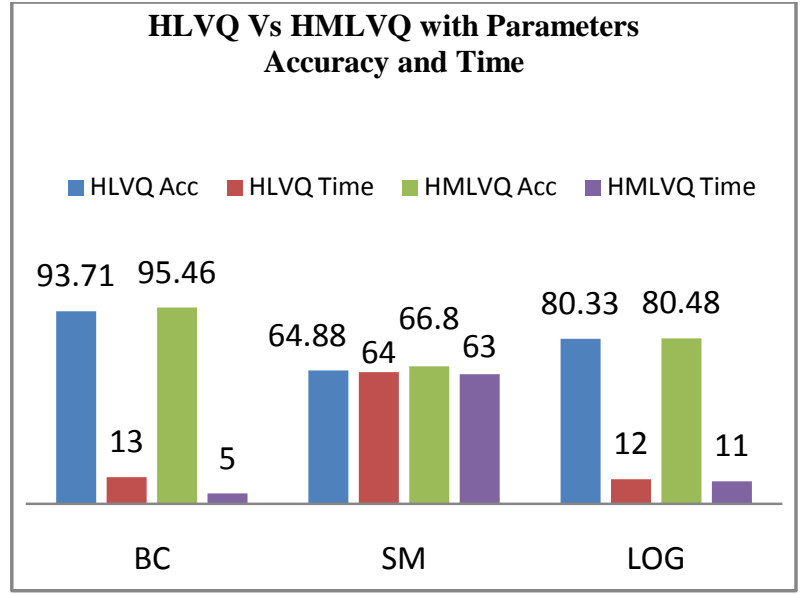

\section{CONCLUSION}

At the end, after studying, learning and comparing a lot, to handle the large volume of web data, there would be a definite need of neural network concept, because only through neural network learning algorithms, such huge volume of web data can be handled and applied to any application for knowledge extraction. All neural network learning algorithms have their own advantages and disadvantages. LVQ is a supervised model of SOM and used for giving class label to data. LVQ is capable to summarize large datasets to smaller number of codebook vectors that are useful for visualization and classification. Training rate is so fast compare to any other network like back propagation. The Generated model updated incrementally. Various versions of LVQ are having one or more disadvantage such as LVQ need to be able to generate useful distance measures for all attributes. They are highly dependent on initialization parameters and training. So by using more than one LVQ approach together will give benefits in recovering from demerits of LVQ. HMLVQ is one such technique that provides fast and accurate classification with reduced size of codebooks. So, in short, by using HMLVQ, the classification of large web data will become easy. The basic HLVQ algorithm works on the same approach in all hierarchy generation that will give the same static classification results. When HMLVQ uses more than one approach to classify the data, so the disadvantages of the same method will excluded and only merits of both approaches are enlighten for accurate classification. So, HMLVQ will be proved as a great classification technique and will be useful for huge datasets as well.

\section{REFERENCES}

[1] Sonali muddalwar, Shashank Kawan, "Applying artificial neural networks in web usage mining", international journal of computer science and management research, vol. 1 issue 4 [Nov-12]

[2] Anshuman Sharma, "Web usage mining neural network", international journal of reviews in computing, vol. $9,\left[10^{\text {th }}\right.$ april, 2012]

[3] Valishali A. Zilpe, Dr. Mohammad Atique, "Neural network approach for web usage mining", ETCSIT, published in IJCA [2011]

[4] Jaydeep Srivastava, "Web Mining: Accomplishments and future directions",

[5] http://www.cs.unm.edu/faculty/srivastava.html

[6] John R. Punin, Mukkai S. Krishnamoorthy, Mohammed J. Zaki, "Web usage mining- language and algorithms", rensseluer polytechnic institute, troy NY 12180

[7] Jaykumar Jagani, Prof. Kamlesh Patel, "A survey of web usage mining with neural network and proposed solutions on several issues", ISSN: 09756760, Nov 12 To Oct 13, Volume - 02, Issue [02]

[8] Renata M. C. R. de Souza, Telmo de M. Silva Filho," Optimized Learning Vector Quantization Classifier with an Adaptive Euclidean Distance", 19th International Conference, Limassol, Cyprus, September 14-17, 2009, volume 5768

[9] Diamantini, Claudia, Spalvieri, A. "Certain facts about Kohonen's LVQ1 algorithm", Circuits and Systems, 1994. ISCAS '94., 1994 IEEE International Symposium on (Volume:6 )

[10] Sang-Woon Kimy and B. J. Oommenz, "Enhancing Prototype Reduction Schemes with LVQ3-Type Algorithms", Natural Sciences and Engineering Research Council of Canada, and Myongji University, Korea, kimsw@mju.ac.kr, oommen@scs.carleton.ca

[11] R. R. Janghel, Ritu Tiwari, Anupam Shukla," Breast Cancer Diagnostic System using Hierarchical Leaming Vector Quantization", IJCA Proceedings on National Seminar on Application of Artificial Intelligence in Life Sciences 2013

[12] Mahesh kumar, Uday Kumar," Classification of Parkinson's disease using Lvq, Logistic Model Tree, K-star for Audioset” , Hogskolan Darlana University, 2011, roda wagen 3s-781 88. 